\title{
Alignment System for Full-Shell Replicated X-Ray Mirrors
}

\author{
Mikhail Gubarev ${ }^{1}$, Brian Ramsey ${ }^{1}$, William Arnold ${ }^{2}$, \\ ${ }^{1}$ MSFC/NASA, Huntsville, AL 35812 \\ ${ }^{2}$ Jacobs ESTS Group, MSFC/NASA, Huntsville, AL 35812
}

\begin{abstract}
We are developing grazing-incidence $\mathrm{x}$-ray optics for high-energy astrophysical applications using the electroformnickel replication process. For space-based applications these optics must be light-weight yet stable, which dictates the use of very-thin-walled full-shell mirrors. Such shells have been fabricated with resolution as good as 11 arcsec for hard $\mathrm{x}$-rays, and technology enhancements under development at MSFC are aimed at producing mirrors with resolution better than 10 arcsec. The challenge, however, is to preserve this resolution during mounting and assembly. We present here a status report on a mounting and alignment system currently under development at Marshall Space Flight Center designed to meet this challenge.
\end{abstract}

Keywords: high-energy x-ray optics, high-resolution x-ray optics, electroform-nickel replication, x-ray optics alignment

\section{INTRODUCTION}

Revolutionary advances to the field of the soft x-ray astronomy have been brought by the use of focusing $\mathrm{x}$-ray optics. To carry this revolution forward within manageable budgets requires technologies capable of fabricating light weight $x$ ray mirrors that can be nested to give large collecting area. One of these processes is electroform nickel replication (ENR), in which thin nickel mirror shells are plated onto figured and superpolished aluminum mandrels and subsequently released by differential thermal contraction. The ENR process has been successfully used to produce x-ray mirrors on previous missions such as Swift and XMM-Newton. ENR mirror shells are full circles of revolution so the mirrors are stable and can be self-supporting. Moreover, since the mirror shells are replicated from mandrels with a monolithic structure containing both parabolic and hyperbolic segments, the alignment of the individual segments to each other is avoided. Recent improvements in the mirror shell replication process at Marshall Space Flight Center (MSFC) have led to fabrication of individual mirror shells up to a factor of four times thinner than the thinnest shells used on XMM and with 11-15 arc seconds half power diameter (HPD) angular resolution at $40 \mathrm{keV}$.

To date, the largest error component in our x-ray optics arises from shell mounting. For example, we have developed 100-micron-thick nickel shells for the hard-x-ray telescope on Constellation- $X^{1}$ (now IXO). These shells typically have angular resolutions of better than 15 arcsec HPD when free standing (derived from metrology), but when mounted we measure 20-30 arcsec . Another example is the mirror modules developed for our HERO balloon payload. The 250micron-thick HERO ${ }^{2}$ mirror shells have measure angular resolutions of 11-15 arcsec, but with the finished modules we achieve 20-25 arcsec. The mounting in these cases is a significant challenge because very-thin shells are easily distorted under contact with mounting hardware or through shrinkage of attachment epoxy. Improvements in our process, to reduce mounting distortions, would benefit the two missions above, although improved resolution over that achieved is not a hard requirement.

Our immediate need for improved mounting is, however, to satisfy the requirements of the FOXSI solar x-ray rocket payload, a Berkeley-led mission with MSFC-produced optics. The goal here is to achieve 15 arcsec HPD module angular resolution, or better, with $0.25-\mathrm{mm}$-thick nickel shells. Beyond this, future mission demand even greater angular resolutions. For example, several potential future missions, such as the Wide Field X-Ray Telescope ${ }^{3}$, and XENIA ${ }^{4}$, require x-ray optics with angular resolutions of 10 arcsec or better, and this places stringent requirements on the mounting and alignment schemes utilized in such applications. Thus we are developing an alignment and mounting system to meet the challenges of current and future flight payloads. 


\section{SHELL ALIGNMENT AND MOUNTING STATION}

The support structure of a full shell $\mathrm{x}$-ray telescope would consist of a housing tube with two precision-fabricated spiders attached to opposite ends of the tube. The inner hubs of the spiders may also be connected with each other by a central column. The central column, the spiders and the housing are made from materials with a coefficient of thermal expansion matched closely to the nickel alloy of the shell mirrors. Each spider would have radial spokes to hold the individual shells. The overall geometry of the telescope module is shown in figure 1.

A full-cylinder-shell mirror alignment and mounting station is currently under development at MSFC. The picture of the alignment and mounting station with a $230 \mathrm{~mm}$ diameter shell installed is shown in figure 2 . The design considerations for the station were discussed in a previous paper $^{5}$. It was shown that mirror shell distortions due to tilt and support point displacements can be determined using shell circularity measurements carried out close to the shell edges. Ideally the measurements have to be done on the inside of the shell mirror and such a possibility is considered for large diameter shell mirrors. For hard $\mathrm{x}$ rays mirrors which typically have small diameters, the circularity measurement has to be carried out on the outside of a shell with a correction made for local shell thickness variation, and these measurement can be done using non-contact sensors. The non-contact displacement sensors used for the circularity measurements are Keyence laser triangulation probes with a resolution of 10 $\mathrm{nm}$ and accuracy better than $60 \mathrm{~nm}$. The alignment station, when completed will be integrated with a Centroid Detector Assembly (CDA), available at MSFC, which will permit the Hartmann test for the final mirror assembly. ${ }^{6}$

The station consists of a base plate assembly, a circularity measurement system, a spider support assembly and a computer-controlled shell support and positioning system. The positioners, the controllers and the measurement systems interface with the control computer (PC). Custom software has been developed to control the shell positioners and to collect and analyze the circularity data in order to determine the optimized algorithm for the shell alignment.

The base plate assembly consists of a motorized rotary air-bearing table with a circular base plate. A central column assembly is mounted on the plate. The column is being designed to support the top spider assemblies. The column can be easily removed from the base plate

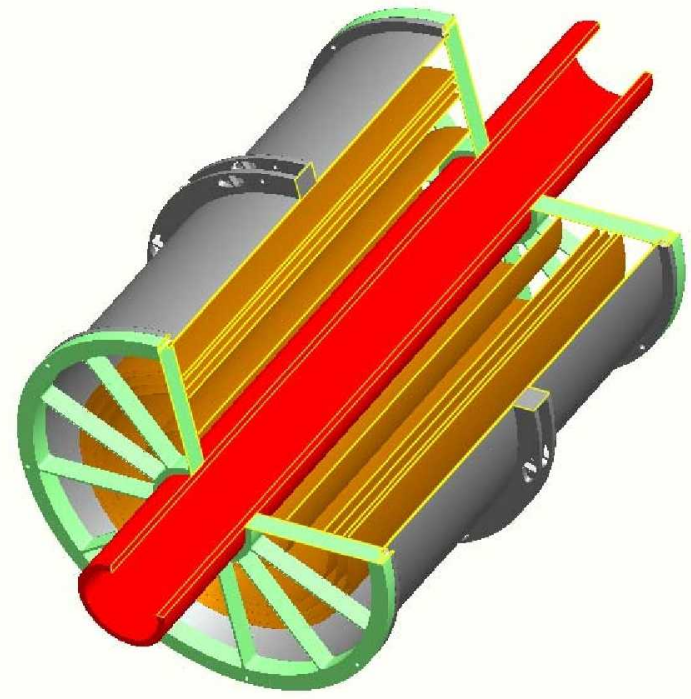

Figure 1. A schematic representation of an $x$-ray telescope module. For simplicity only five mirror shells are shown. The actual number of spider spokes to support the nested mirror shells is variable and determined through finite element analysis.

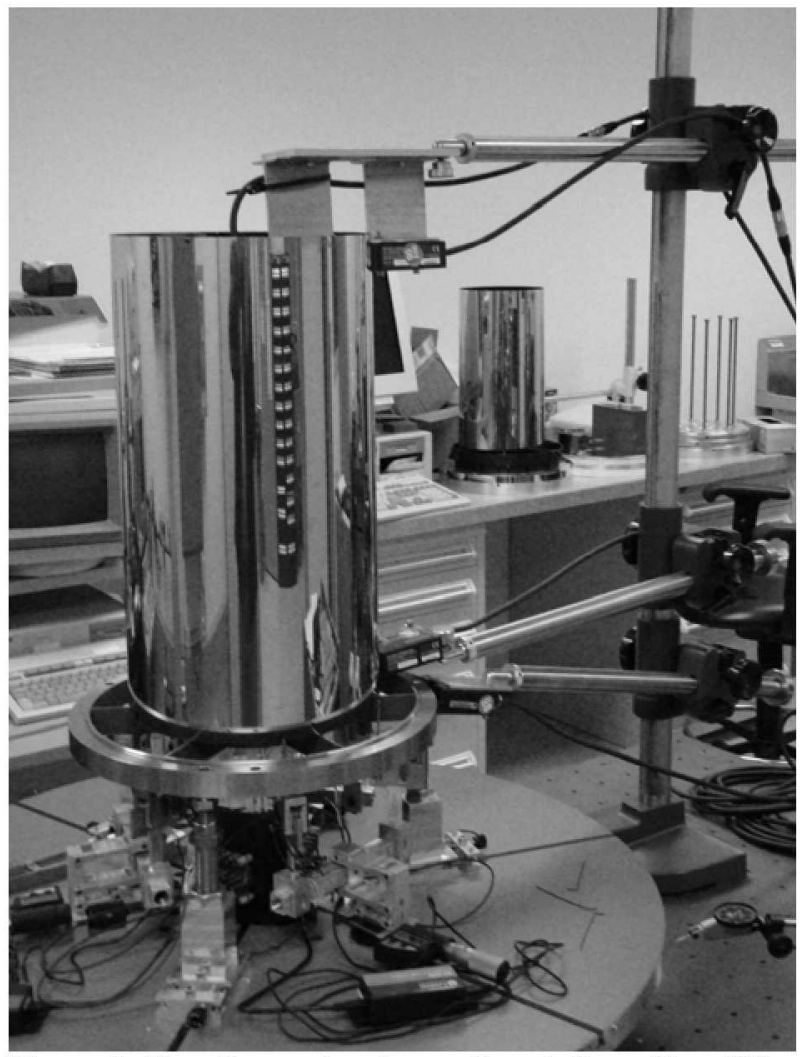

Figure 2. The alignment and mounting station with a 230 $\mathrm{mm}$ diameter shell under alignment. 


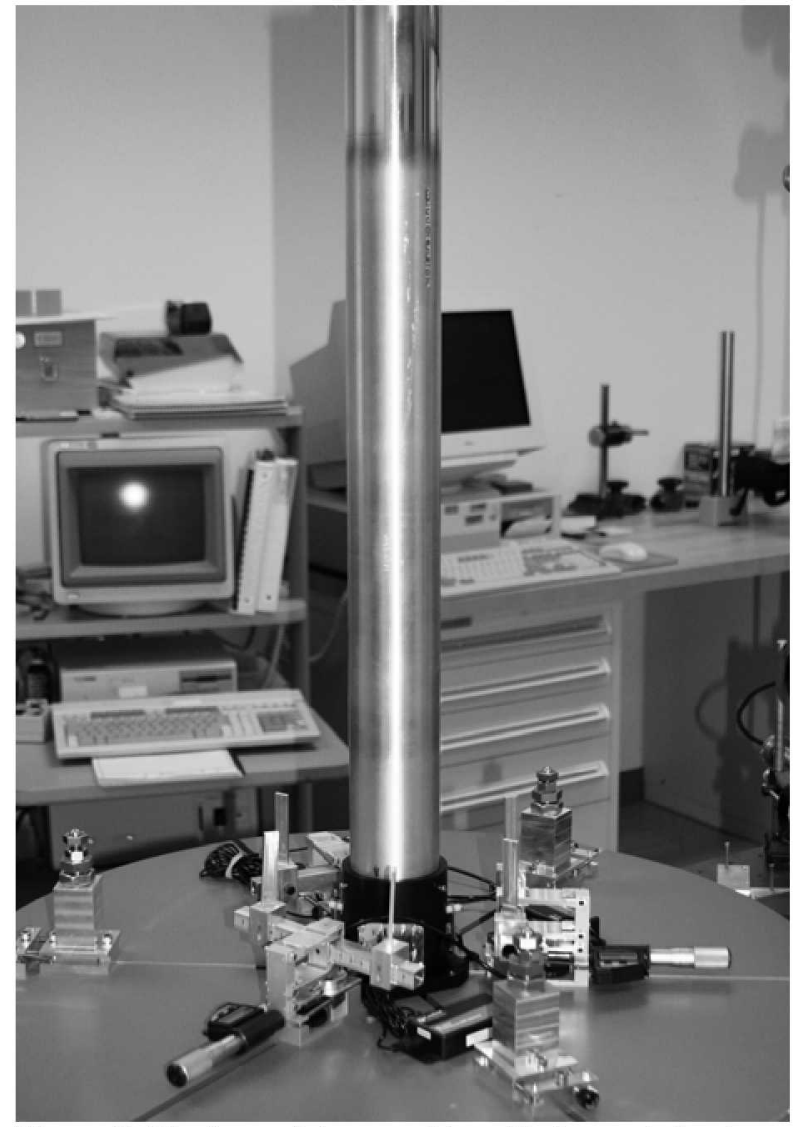

Figure 3. The base plate assembly with the central column installed. The top part of the column is polished to improve the accuracy of the column circularity measurement needed to control the wobble of the rotary table during the shell alignment procedure. assembly or changed to smaller diameter column, if it is needed. The edge of the base plate and the top part of the central column were diamond turned and polished to permit accurate measurement using the Keyence probes. These measurements are used to monitor the wobble of the rotary table during the shell circularity measurements. A slip-ring assembly is attached to the plate to provide the power and a feedback to the actuators which control the radial and axial positions of the mirror shell under alignment. Currently about half of the slip ring communication channels are committed to the elements of the support and positioning system, the rest of the channels are reserved for future positioning system to control top of the shell if such an option is needed.

The metrology system consists of a sensor support stand with non-contact displacement Keyence sensors controlled by the computer. The Keyence sensors are used to measure the mirror shell circularity and azimuthal shell thickness variations profiles needed to permit the circularity measurement on outside of the shell. The circularity, de-centering and the tilt of the mirror is derived from the measurement results using the thin-shell finite-element model and the software we have developed. This information is used to derive the form and position of the shell being aligned and to define the alignment strategy. For shell thickness variations the Keyence sensors can be fitted inside of a shell with inside diameter not smaller than $130 \mathrm{~mm}$. We are planning the use of our Vertical Long Trace Profiler (VLTP) to obtain shell thickness variation profiles for shells with smaller inside diameters.
The shell support and positioning system consists of two motion-independent subsystems: an axial support system and a radial positioning system. The two systems are kept independent to simplify the alignment algorithms.

The axial support subsystem is multi-point reconfigurable whiffle tree kinematic system designed to hold the shell under alignment at the bottom. The axial support subsystem permits the shell weight to be distributed over the required number of support points. These support points are grouped so the tilt and the height of the system can be adjusted kinematically, i.e. through only three axial positions adjusters. Each of the three axial actuators has a load cell to measure the shell weight and to control how uniformly the shell weight is distributed over support points. Depending on the shell weight and diameter the axial support subsystem can be configured to have a different

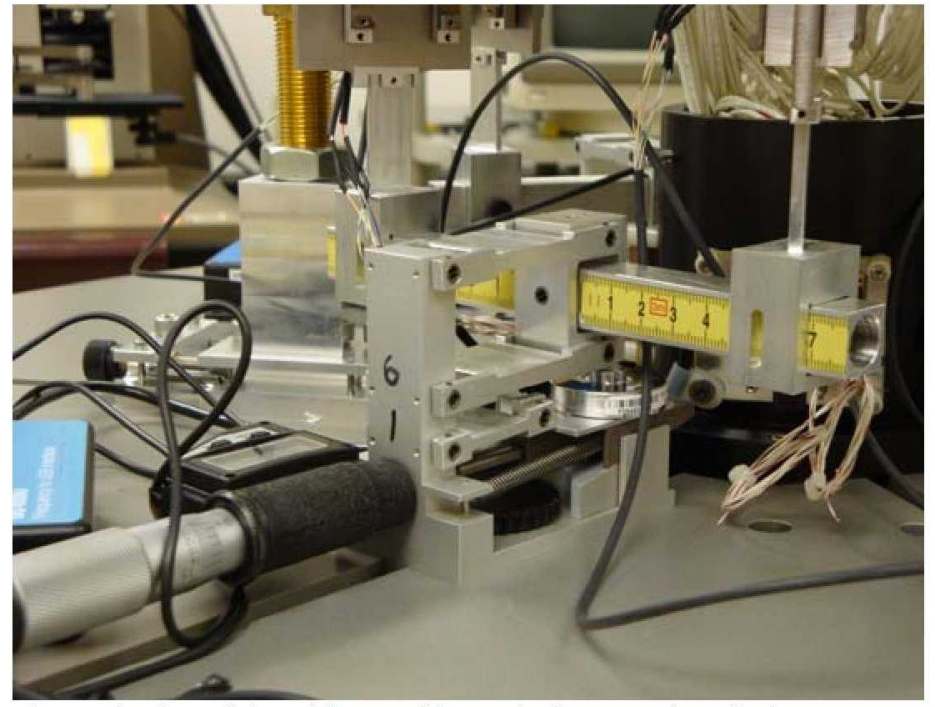

Figure 4. The axial positioner with two inch worms installed 
number of support points from three to 24. A picture of the base plate assembly with the central column, the spider support positioners and the axial support subsystem configured for six support points is shown in figure 3. A close up picture of the axial positioner is shown in figure 4.

The radial positioning subsystem includes the radial slides located at the bottom of the shell and custom built inch-worm actuators. The inch-worm actuators have three piezo stacks. Two piezos control two clamps mounted on the radial slide and the third actuator is attached to both clips. By tightening and releasing the clips the actuator can adjust the local radial position of the shell. An inch worm actuator supporting a mirror shell under alignment is shown in figure 5 .

The spider support assembly includes bottom and top spiders connected by the module column and the bottom spider positioning stages. The bottom spider has a monolithic structure and it is supported by three stages installed at the base plate. The bottom spider positioning assembly is shown in figure 6 . The stages radial position and height can be adjusted to position and level the bottom spider. The inner hub of the top spider is connected to the upper part of the module column. The alignment and mounting process starts with the innermost shell and works outward with the mirror shell epoxied first to the bottom spider, then, to the top spider. To allow nesting the shells together into the telescope module the top spider is designed to be build incrementally using sectional spoke

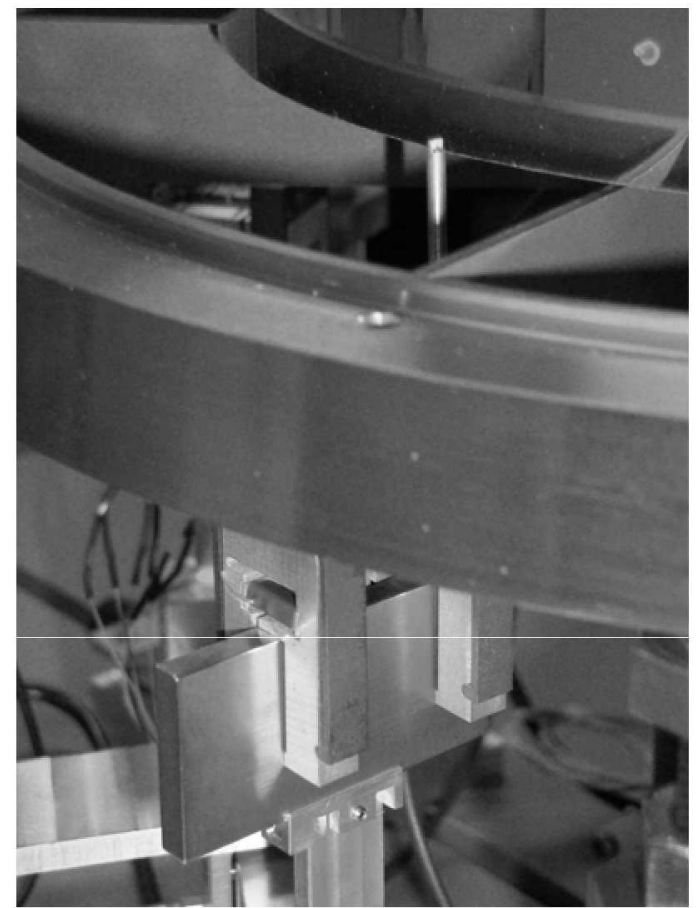

Figure 5. An inch worm actuator with a radial slide positioned under the bottom spider. The shell is supported by a tall rod attached to the inch worm. extensions as it shown in figure 7. In this design, the main structural stiffness and load path is provided by the bottom spider. The function of the top spider is to supply lateral support and control mode shapes in the shell. To connect a mirror shell to the bottom spider a shell attachment clip with two perpendicular grooves will be used. A schematic of the shell attachment clip is shown in figure 8. The clip would be positioned near the shell edge such that the gaps between the shell and the walls of the clip groove are equal and then epoxied to the shell. After the shell is aligned the clip can be epoxied to the spider spoke. In this case the bonding stresses would mainly be acting tangentially to the shell and so shell figure distortions would be minimized.

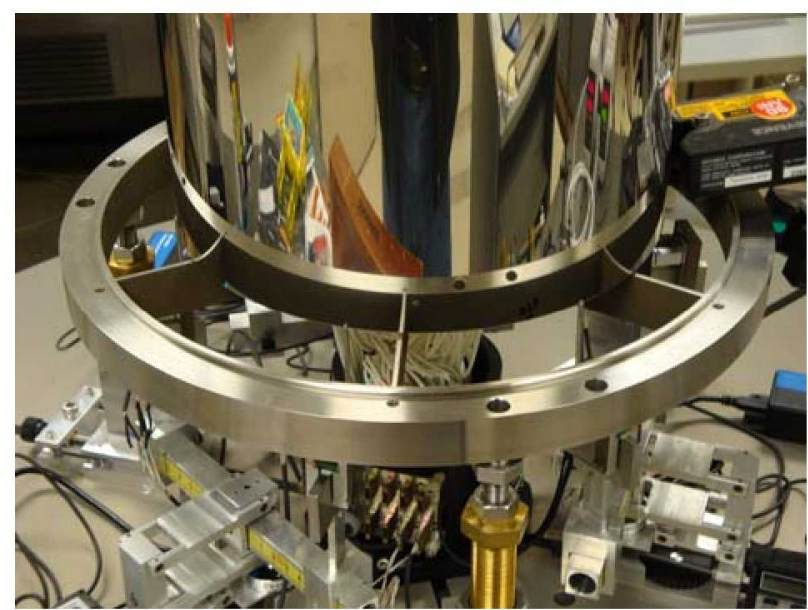

Figure 6. The bottom spider with the mirror shell held above it during alignment experiments.

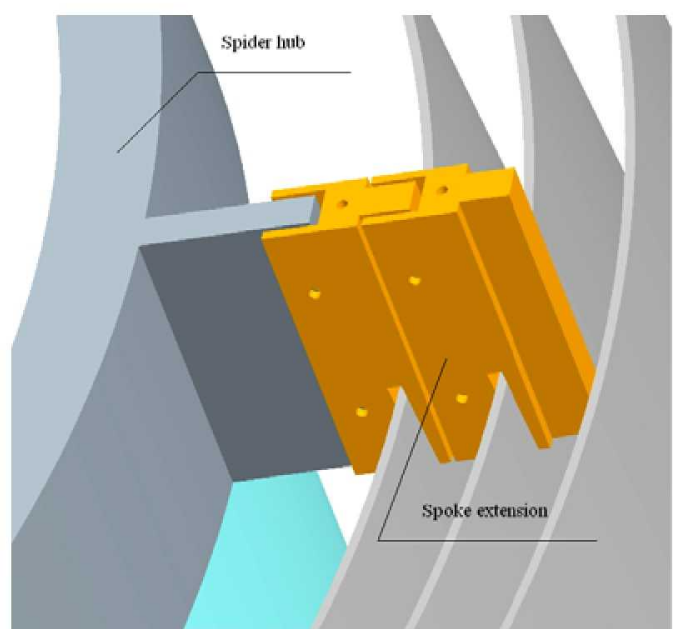

Figure 7. Top spider design 
The station has been used to measure the variation in thickness of shells, which will subsequently be mounted, to verify the accuracy of this approach. For this, the mirror was installed onto a six point waffle-tree support system. Three non-contact displacement sensors were installed, two at the top of the mirror, aligned with each other inside and outside of a shell, and the third one at the bottom of the shell. For this measurement the central column that is used monitor the wobble of the rotary table during the shell alignment procedure was removed to allow the sensor to fit inside the shell.

In the thickness measurements one of the top and the bottom sensor were used to measure to correct the global tilt of the shell mirror and to center the shell relatively to the vertical axis of the rotary table. Then, the two top sensors were used to measure the thickness variation of the shell mirror at a point $10 \mathrm{~mm}$ down from the shell edge. Then, the shell was flipped and the procedure was repeated for the opposite end of the mirror. A sample of the shell thickness variation measured is shown in figure 9.

\section{CONCLUSIONS AND FUTURE WORK}

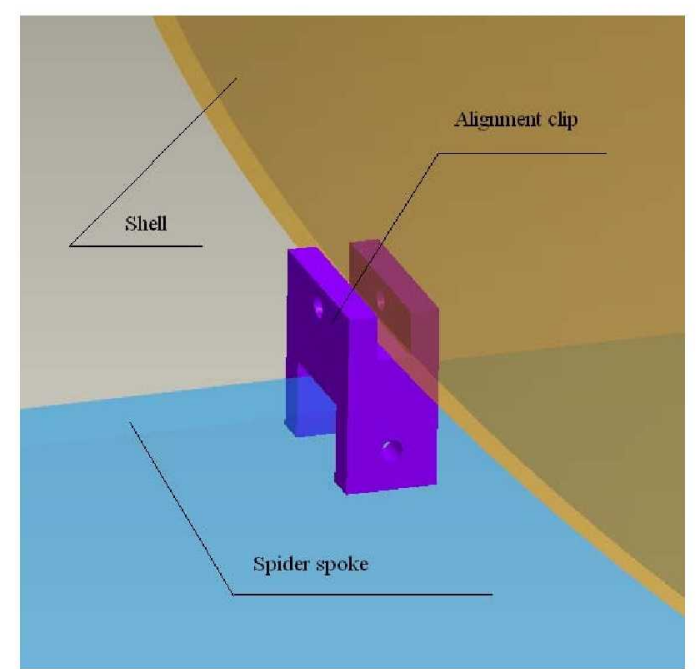

Figure 8. A schematic of the shell attachment clip. The clip is pre-glued to the shell. After the shell is aligned the clip is glued to the spider spoke, so the bonding forces are directed azimuthally.

All major components of the alignment and mounting station, and the software developed to control the station, have been built and successfully tested. Currently, efforts are focused on testing the accuracy of the system and testing the procedures that were outlined previously ${ }^{5}$.

The alignment and mounting station described here was designed for a range of shell diameters from 100 to $500 \mathrm{~mm}$ with shell lengths about $600 \mathrm{~mm}$. The mirror shells for the FOXSI solar x-ray rocket will have a smaller range of diameters $(70$ to $100 \mathrm{~mm})$ with similar shell length. To accommodate the smaller shell diameters, it will be necessary to make a few modifications to the alignment and mounting station. These modifications consist of substituting a smaller central column of 1.5-2.00" diameter for the current 4.00" diameter part. The central column is used to establish the axis of rotation to the accuracy levels necessary for aligning the shell stack to a common axis and to hold the

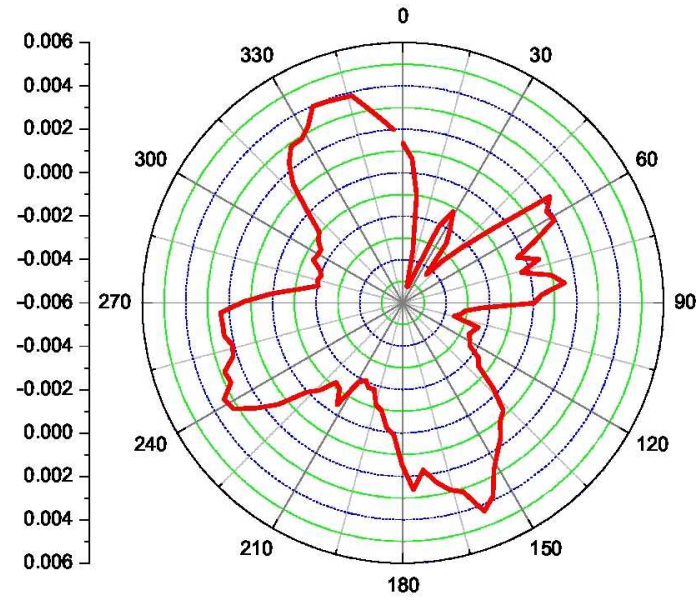

Figure 9. A sample of the shell thickness variation (in millimeters) profile measured using the alignment station. connection wires reserved for the top alignment system. The baseline FOXSI support spiders will be designed to accommodate a central hole of approximately 2.00 " diameter.

Once the shells are aligned and bonded to the bottom support spider, the outer mirror cell tube can be fitted and the other aperture plate positioned over the shells. The FOXSI mirror shells are relatively stiff, so once the alignment is verified by the Keyence probes, the upper shells can be bonded to the top spider.

The final modification required is to position the inchworms into such small diameter spaces. A redesign of the "whiffle" tree interface to the inch worm is possible, but for the smaller shells a completely different approach may be required. We are currently evaluating a means to cantilever the 
radial position mechanisms (the inch-worms on the original system) and an alternative actuator.

The three main axial assemblies will remain the same, with some adaptor attached. The axial units provide precision tilt and axial position adjustment to the shell. We are evaluating two concepts for the radial positioner, one based upon a relatively inexpensive micro stepper lead screw component and reduction mechanism and the other, a direct micrometer drive design.

\section{REFERENCES}

${ }^{1}$ Ramsey, B. and Gorenstein, P., “A Hard-X-Ray Telescope Science Enhancement Package for the Constellation-X Mission," SPIE Volume 6688, 2007.

${ }^{2}$ M. Gubarev, C. Alexander, B. Ramsey, "Alignment, assembly, and testing of high-energy x-ray optics" Proc. SPIE, Vol. 5900, 59000T (2005);

${ }^{3}$ Murray, S., et al., 'The Wide Field X-Ray Telescope Mission,' SPIE volume 7011, 2008.

${ }^{4} \mathrm{http}: / / \mathrm{sms} . \mathrm{msfc}$.nasa.gov/xenia/

${ }^{5}$ M. Gubarev, W. Arnold, C. Benson, T. Kester, D. Lehner", B. Ramsey, R. Upton" "Mounting and alignment of fullshell replicated x-ray optics", Proc. SPIE, 6688, pp. 66881F, 2007.

${ }^{6}$ Glenn P., "Centroid Detector Assembly for the AXAF-I Alignment Test System”, Proc. SPIE, 2515, 352, 1995 


\section{Alignment System for Full-Shell Replicated X-Ray Mirrors}

\section{Mikhail Gubarev', William Arnold ${ }^{2}$, Brian Ramsey ${ }^{1}$}

${ }^{1}$ MSFC/NASA, Huntsville, AL 35812

${ }^{2}$ Jacobs ESTS Group, MSFC/NASA, Huntsville, AL 35812 


\section{Need for Full Shell Mirror Alignment System}

In many applications errors in the mounting of thin shells limits the overall optics performance.

electroform nickel replication process has demonstrated $\mathrm{x}$-ray mirrors with angular resolution better than $10 \mathrm{arc}$. Sec HPD. Mounted nickel mirror shell telescopes have angular resolution $\sim 20$ arc. Sec. HPD

Missions to benefit:

IXO HXT

FOXSI

Xenia

WFXT

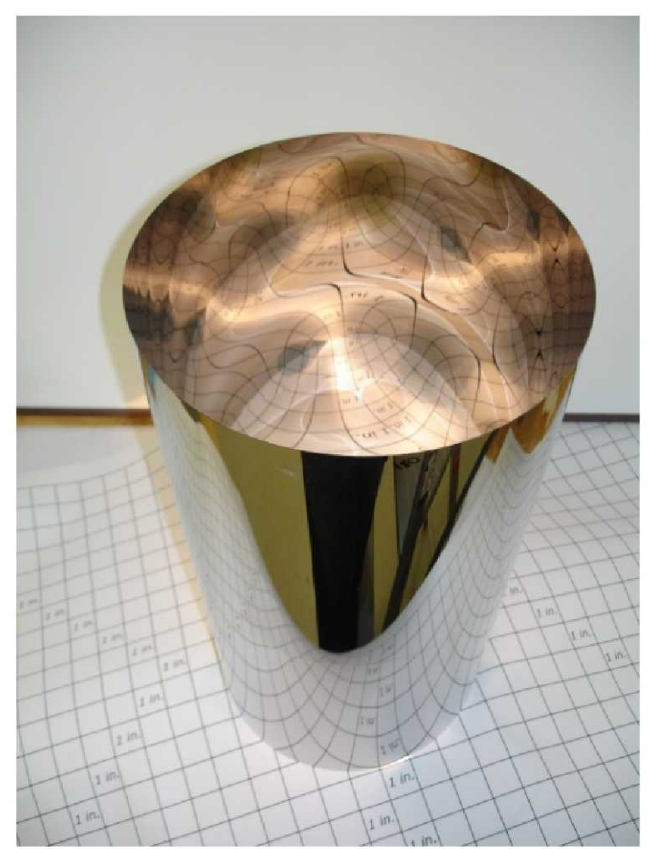

$23 \mathrm{~cm}$ diameter, $10 \mathrm{~m}$ focal distance x-ray mirror for the IXO HXT 


\section{Full Mirror Shells}

Advantages:

Sturdy, (well, relative to segmented optics); The form of the shell can be predicted, if the displacements are known;

Fewer optical elements;

Disadvantages:

Metrology is challenging, especially during mounting and alignment

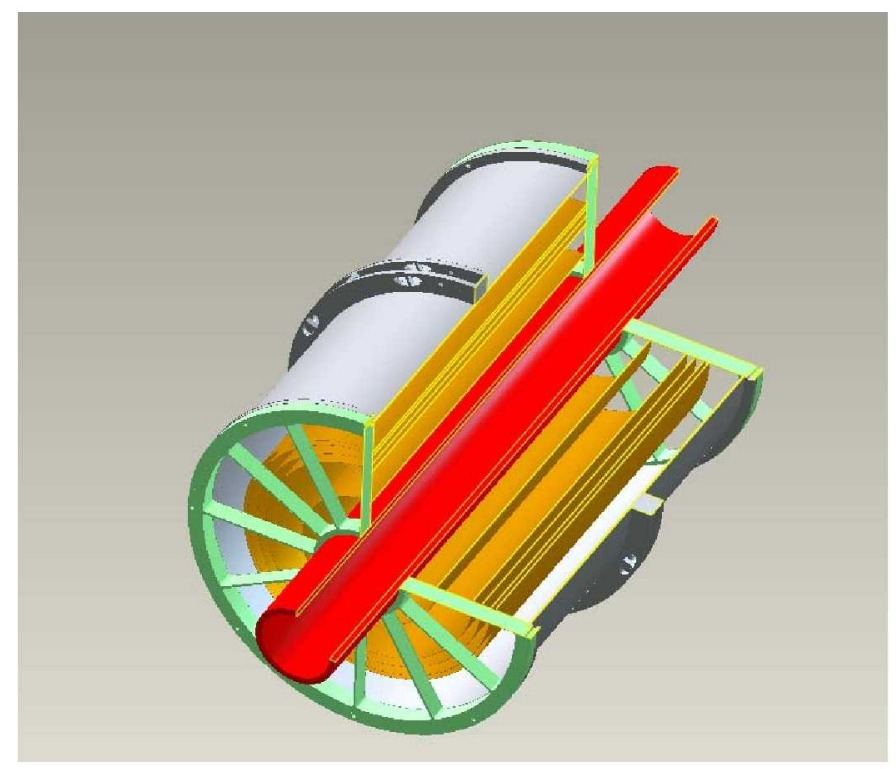

A schematic representation of the $x-$ ray telescope module. 


\section{Full Mirror Shells}

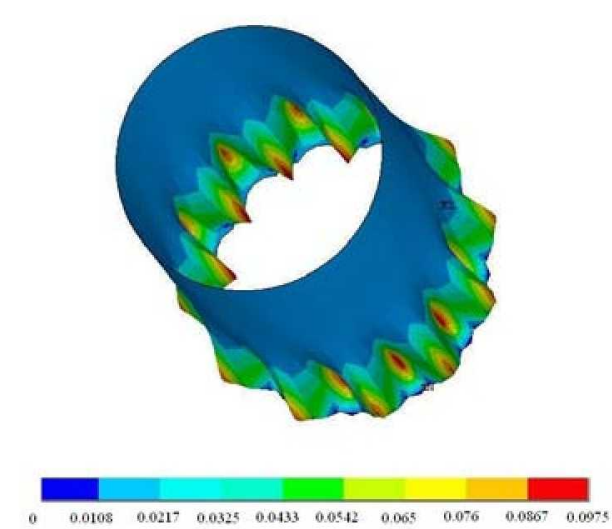

Shell supported with 12 points at the bottom.

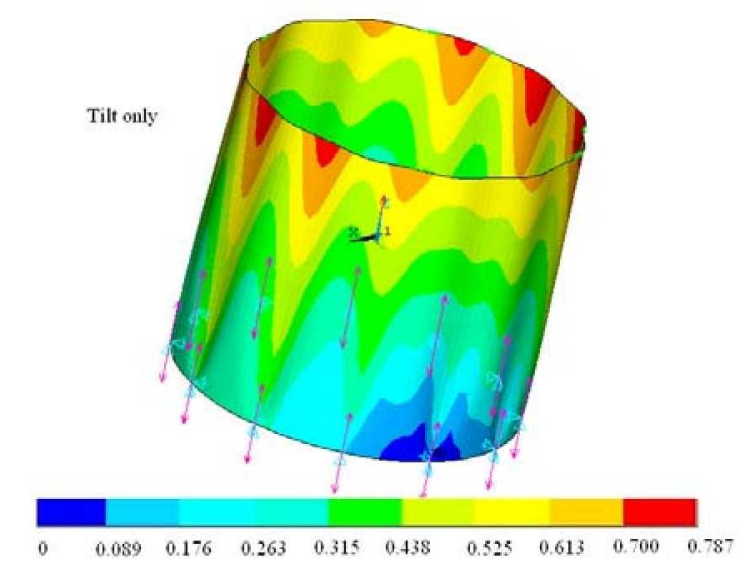

The shell is tilted by 1 microradian.

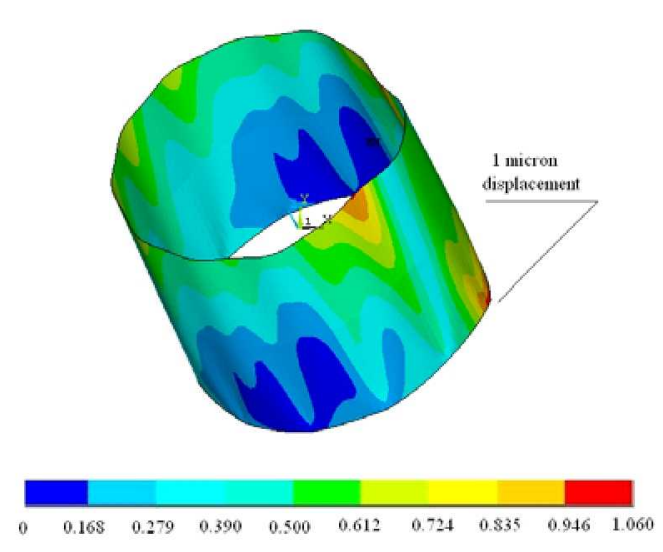

The shell is tilted by 1 microradian. One of the support points is displaced by 1 micron

If mirror shell misalignment is relatively small, the deformations due to the support structure are localized and the form of the shell can be judged from circularity measurements 


\section{SHELL ALIGNMENT AND MOUNTING STATION}

Philosophy:

Using high precision non-contact position sensors monitor circularity of the shell at least at two positions - very top and very bottom.

Utilize custom inch worm actuators to position the shell with submicron accuracy Use multiple point waffle tree to support the shell

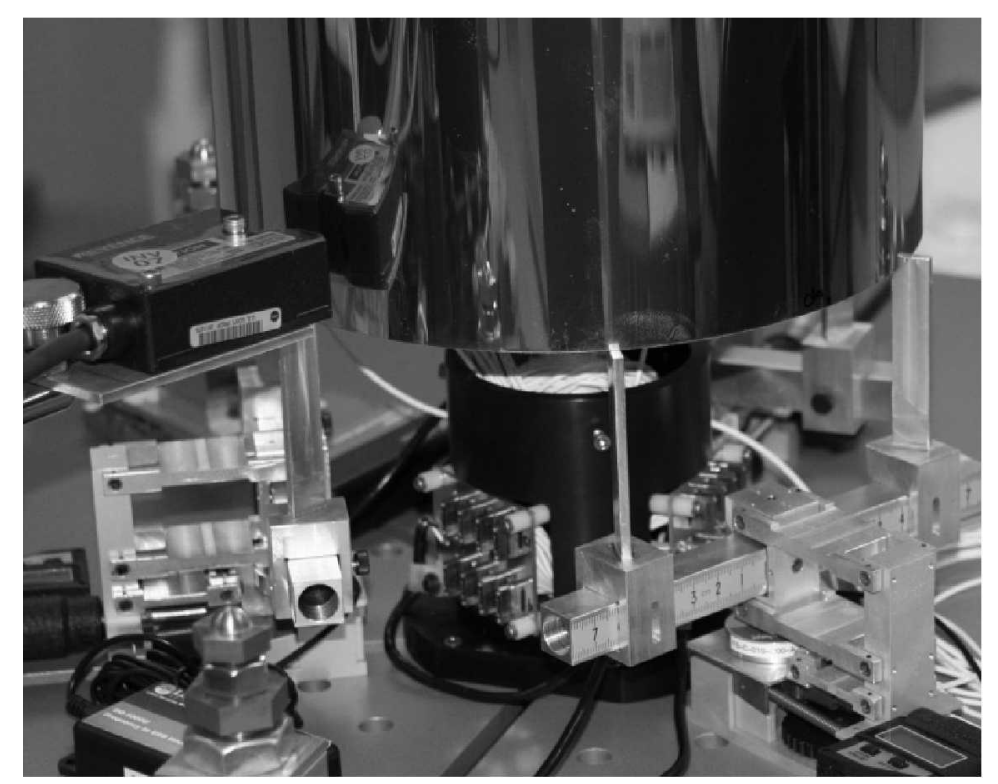

The non contact displacement sensor (on left) is placed at the bottom of the shell mirror installed on six points waffle- tree kinematic system. 


\section{SHELL ALIGNMENT AND MOUNTING STATION}
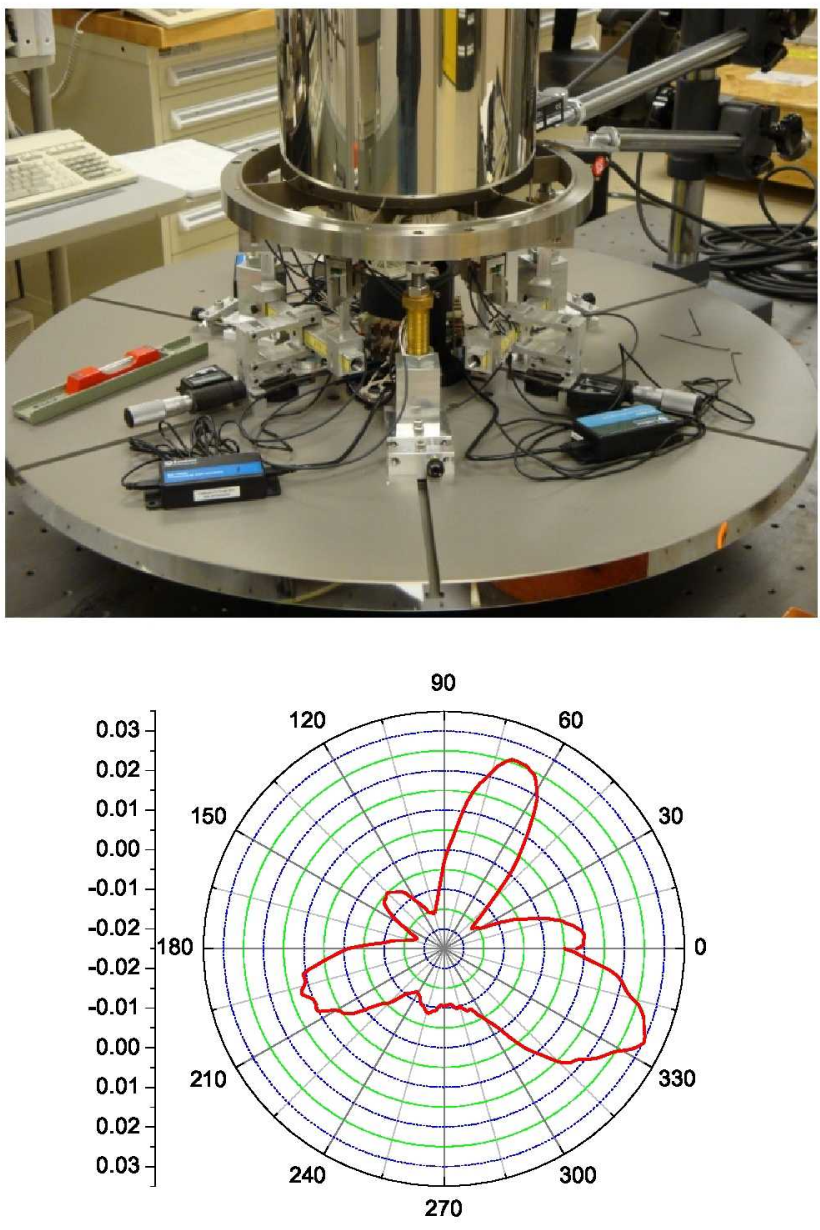

A sample of the thickness variation (in millimeters) profile measured using the alignment station
A mirror shell during alignment procedure installed above spider

Waffle tree support point with the inch worm actuators

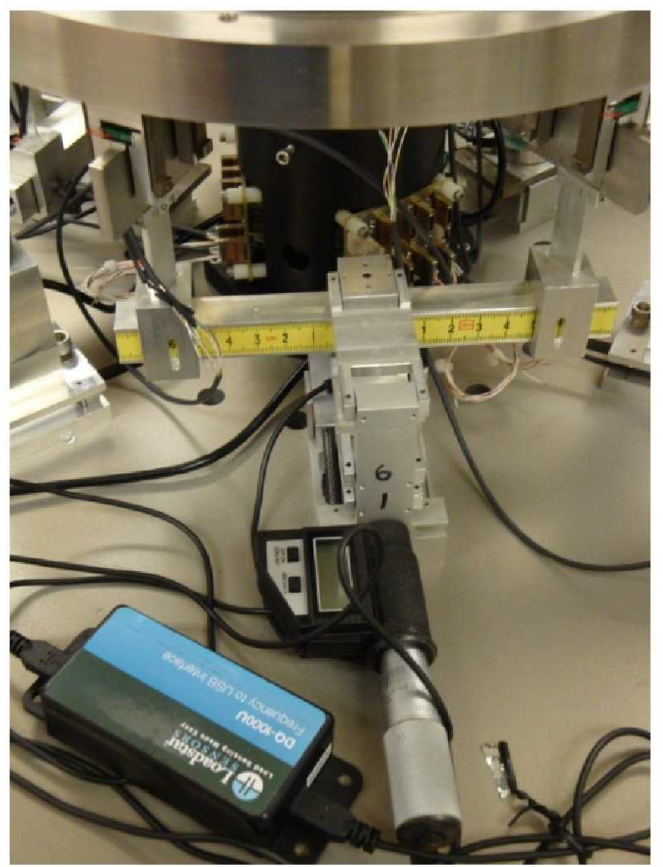

All subsystems of the alignment and mounting system have been tested. Currently, the shell alignment experiments underway at the MSFC 


\section{Conclusions}

The mounting and alignment system is designed to assemble very-thin full shell mirrors into x-ray telescope module without significant angular resolution degradation.

High precision non-contact position sensors are used to monitor the shell distortions during the alignment

The shell positioning is done through custom build piezo inch worm actuators.

All subsystems of the alignment and mounting system have been tested.

Currently, shell alignment experiments are underway at MSFC 